\title{
Phase Coexistence of Cluster Crystals: Beyond the Gibbs Phase Rule
}

\author{
Bianca M. Mladek, ${ }^{1}$ Patrick Charbonneau, ${ }^{2}$ and Daan Frenkel ${ }^{2, *}$ \\ ${ }^{1}$ Center for Computational Materials Science and Institut für Theoretische Physik, Technische Universität Wien, \\ Wiedner Hauptstrasse 8-10, 1040 Wien, Austria \\ ${ }^{2}$ FOM Institute for Atomic and Molecular Physics, Kruislaan 407, 1098 SJ Amsterdam, The Netherlands
} (Received 17 August 2007; published 7 December 2007)

\begin{abstract}
We report a study of the phase behavior of multiple-occupancy crystals through simulation. We argue that in order to reproduce the equilibrium behavior of such crystals, it is essential to treat the number of lattice sites as a constraining thermodynamic variable. The resulting free-energy calculations thus differ considerably from schemes used for single-occupancy lattices. Using our approach, we obtain the phase diagram and the bulk modulus for a generalized exponential model that forms cluster crystals at high densities. We compare the simulation results with existing theoretical predictions. We also identify two types of density fluctuations that can lead to two sound modes and evaluate the corresponding elastic constants.
\end{abstract}

DOI: 10.1103/PhysRevLett.99.235702

At finite temperatures, all crystals contain point defects. This means that the ratio between $N$, the number of particles, and $N_{c}$, the number of unit cells is not fixed by geometry. In the language of Ref. [1], $N_{c}$ is a "constrained" thermodynamic variable. A general variation of the Helmholtz free energy for a one-component crystal can be written as

$$
d F=-S d T-P d V+\mu d N+\mu_{c} d N_{c},
$$

where $S$ is the entropy, $T$ the absolute temperature, $P$ the pressure, $V$ the volume, $\mu$ the chemical potential of the constituent particles, and $\mu_{c}$ the "cell chemical potential" conjugate to the number of unit cells. If $N_{c}$ is free to change, it will take on a value such that $\mu_{c}=0$ to minimize the system's free energy; hence, its value is a function of $N, V$, and $T$. While in a bulk equilibrium crystal this can be realized by making adjustments at surfaces, interfaces, and boundaries, in a simulation box or in a quenched sample the initial lattice geometry constrains the system. In simple crystals, however, the equilibrium concentration of point defects is usually so low that their effect on the phase behavior is negligible [2,3]. For instance, at melting, the chemical potential of a hard-sphere crystal with vacancies roughly differs by as little as $10^{-3} k_{B} T$ from that of a defect-free crystal for which $N_{c}=N$ [3].

Interestingly, the situation is dramatically different for systems that form hypercrystals, such as certain liquid crystal phases [4], quantum Hall effect bubble solids [5,6], or, as in this Letter, "cluster crystals" [7-12]. These unusual crystalline materials can have a number of particles per lattice site much larger than 1 . These last solids form in systems of particles that interact via a bounded, short-ranged, and purely repulsive pair potential whose Fourier transform has negative regions, as has been anticipated for amphiphilic dendrimers [13]. The effect of $\mu_{c}$ on the phase behavior then becomes all important, which has profound consequences for the numerical study
PACS numbers: $64.70 . \mathrm{Kb}, 64.70 . \mathrm{Dv}, 82.30 . \mathrm{Nr}, 83.80 . \mathrm{Rs}$

of their phase transitions. The reason is that in almost all simulations involving crystals, the average number of particles per unit cell is fixed at the outset of the simulation. After that, a change in the density $\rho \equiv N / V$ of the system may still change $P$ and $\mu$ but, as the ratio $N / N_{c}$ is fixed, $\mu_{c}$ will in general not be zero. Hence, conventional simulations do not probe the lowest free-energy state of the crystal. At constant $P$ and $T$, a small variation in Gibbs free energy $G \equiv F+P V$ is of the form $\mu d N+\mu_{c} d N_{c}$. If we fix the ratio $n_{c} \equiv N / N_{c}$, then both $\mu$ and $\mu_{c}$ are constant, so we can integrate to obtain

$$
G=N \mu+N_{c} \mu_{c}
$$

and hence

$$
N_{c} \mu_{c}=F+P V-\mu N .
$$

For a given $N, V, T$, and $N_{c}$, we can use Monte Carlo (MC) simulations to compute $F, P$, and $\mu$ [14]. As all quantities on the right-hand side of Eq. (3) can be determined numerically, while $N_{c}$ is known, we can also compute $\mu_{c}$. This is important because the condition for phase coexistence involving cluster crystals requires equality of $\mu, P$, and $T$ in the coexisting phases and of $\mu_{c}=0$ in all crystalline phases. This latter condition is not normally considered in the discussion of the Gibbs phase rule. However, in his original formulation, Gibbs does allow for the possible existence of other thermodynamic "fields" in addition to $\mu, P$, and $T$ [15].

As an application of this approach, we consider the numerical determination of the phase diagram for the generalized exponential model (GEM- $n) \Phi\left(r_{i j}\right)=\varepsilon e^{-\left(r_{i j} / \sigma\right)^{n}}$ with $n=4$, where $\varepsilon$ and $\sigma$ determine, respectively, the energy and the length scales. For convenience, we set them to unity and consider only reduced units from this point forward. For $n>2$, this system is known to form cluster solids at high densities $[7,8,11,16]$. Its phase diagram is known qualitatively, but not quantitatively: at high $T$, the 
fluid first freezes into a multiply-occupied bcc phase that transforms into a multiply-occupied fcc phase at higher densities. By contrast, upon compression at low $T$, the system undergoes a "normal" freezing transition to a single-occupancy fcc crystal; clustering only sets in upon further compression of the solid.

We perform discretized-space constant- $N V T$ MC simulations $[10,17]$ for $2000-5000$ particles in the temperature regime where multiple occupancy of the crystal lattice sites is expected. We determine the value of $\rho_{c} \equiv N_{c} / V$ such that $\mu_{c}=0$ for every $T$ and $\rho$ state point by starting the fixed $N_{c}$ simulations with a reasonable guess for $N$ and $V$ and iterating until the equilibrium values are located. Via the common tangent construction, we obtain the coexistence densities from the resulting free-energy curves. We note that, because the number of particles per lattice site is free to fluctuate, we cannot use the Einstein-crystal method to compute the free energy of the solid $[14,18]$. Rather, we perform a thermodynamic integration from a reference state of ideal-gas particles that move in potential wells centered around the lattice sites [19]. We also note that for bounded potentials, the Widom particle-insertion method provides an efficient tool to determine the chemical potential, even in the dense solid [14,20]. One could even think of performing a kind of Gibbs-ensemble simulation where two systems exchange both particles and volume [21]. However, such a simulation would not locate the correct coexistence point, precisely because the Gibbsensemble method does not impose the condition $\mu_{c}=0$ in all solid phases. That is why we have to follow the rather elaborate route via Eq. (3) to locate the points where the different phases coexist.

In Fig. 1, we compare the $T$ - $\rho$ phase diagram for the GEM-4 with the corresponding density-functional theory (DFT) predictions of Ref. [8]. In the same figure, we also show the estimate of the freezing transition based on the results of simulated annealing MC (SAMC) simulations [10,11]. For the liquid-bcc transition, the liquidus line predicted by DFT is indistinguishable from the "exact" simulation results, and the solid line is only slightly off. The SAMC results, although qualitatively correct, predict a liquid-bcc density gap that is too narrow. This is probably due to finite-size artefacts in Refs. [10,11]. A crucial test of the accuracy of DFT is the prediction of the location of the bcc-fcc transition. As the densities and free energies of these two phases are very close (Fig. 1 lower inset), minor inaccuracies in the theory should have a noticeable effect on the prediction of the transition point. Indeed, we find that even though the DFT free-energy predictions are only off by a small amount (not shown), the location of the phase transition is shifted by roughly $10 \%$ in $\rho$. The $P-T$ phase diagram is shown in the upper inset, where the various state points are accompanied by tangents to the coexistence curves obtained from the simulation free and internal energy results by use of the Clausius-Clapeyron

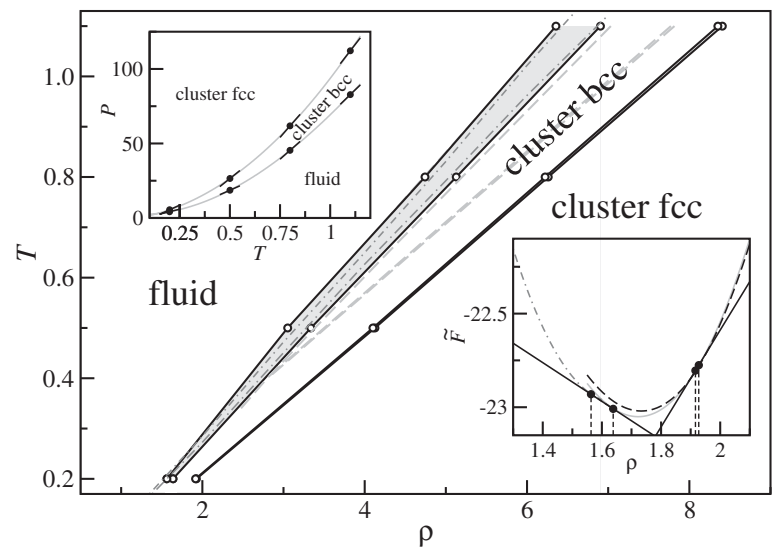

FIG. 1. Comparison of the DFT [8] (dashed lines) and SAMC [10] (dash-dotted lines) $T$ - $\rho$ phase diagrams with the simulation results (points) for the GEM-4. The gray zone highlights the phase coexistence region. Upper inset: $P-T$ simulation phase diagram (points) with the corresponding Clausius-Clapeyron tangents to the coexistence curve (black lines). The solid curves are guides for the eye. Lower inset: shifted free-energy curves $\tilde{F} \equiv F / V T-26 \rho$ (to enhance visibility) at $T=0.2$ for the liquid (dash-dotted gray line), bcc crystal (solid gray line), and fcc crystal (dashed black line), along with the common tangent construction (solid black lines) and the coexistence densities (dots and drop-down lines).

relation [14]. Inspection of the diagram suggests a liquidbcc-fcc triple point around $T_{t} \approx 0.15$, which is much lower than the DFT prediction of $T_{t} \approx 0.4$ [8]. The dramatic shift follows from the small difference between the slopes of the liquid-bcc and the bcc-fcc coexistence curves, leaving the location of the triple point very sensitive to any modification of the latter.

It is interesting to understand the reason for the failure of DFT to predict the location of the solid-solid transition. One of the core consequences of the DFT treatment of Ref. [8] is that the volume of the unit cell in a cluster crystal $v_{c} \equiv V / N_{c}$ is independent of density, so that the average site occupation $n_{c} \propto \rho$. This feature is known to break down in low-density crystals [10] and is also found here to be slightly inaccurate at intermediate temperatures and densities. Though the linear relationship holds, the proportionality is shifted by a constant. As can be gathered from the inset of Fig. 2, this leads to a nonzero value of $\left(\partial v_{c} / \partial \rho\right)_{T}$ for equilibrium states, though the effect vanishes with increasing $\rho$. This suggests that the DFT approximation is asymptotically valid. At intermediate densities, this correction though small might be sufficient to explain the discrepancy between the DFT and the numerical results.

Since thermodynamic equilibrium is only obtained when $\mu_{c}=0$, it would appear that once the equilibrium points are found, all references to the artificial $\mu_{c}$ field can be disregarded. Yet, for quantities involving the second derivative of the constrained free energy, such as the bulk 


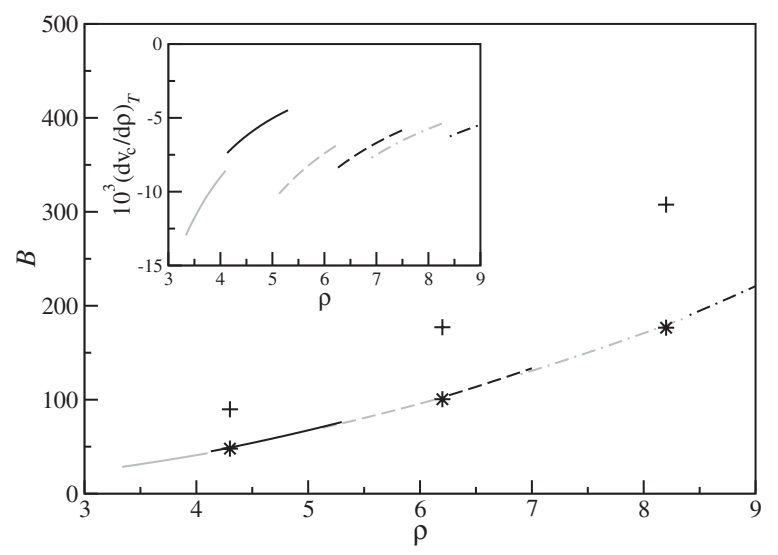

FIG. 2. Bulk modulus results from direct differentiation of the free energy of the stable crystal structures [bcc (gray) and fcc (black) at $T=0.5$ (solid line), $T=0.8$ (dashed lined), and $T=$ 1.1 (dash-dotted line)], along with the values at three state points (stars) computed using Eq. (5). The virial contribution $B_{\text {vir }}$ is shown for reference (crosses). The breakdown of $B$ is also given in Table I. Inset: variation of the lattice volume with density in equilibrium for these same systems. DFT presents this quantity as zero.

modulus $B=V\left(\frac{\partial^{2} F}{\partial V^{2}}\right)_{N, T}$, it cannot be neglected unless one already has the complete equilibrium free-energy curve at hand. In simulations of single-occupancy crystals, $B_{\text {vir }}=$ $-V\left(\frac{\partial P}{\partial V}\right)_{N, T, N_{c}}$ can be computed directly for a given state point through an approach similar to the virial calculation of $P$ [22,23]. For cluster crystals, however, the artificial system conditions further modify the bulk modulus as

$$
\begin{aligned}
B & =-V\left(\frac{\partial P\left(N, V, T, N_{c}^{\mathrm{eq}}\right)}{\partial V}\right)_{N, T} \\
& =B_{\mathrm{vir}}-V\left(\frac{\partial P}{\partial N_{c}}\right)_{N, T, V}\left(\frac{\partial N_{c}}{\partial V}\right)_{N, T, \mu_{c}=0} \\
& =B_{\mathrm{vir}}-\frac{\rho^{2}}{n_{c}}\left(\frac{\partial \mu_{c}}{\partial \rho}\right)_{T, n_{c}}\left[1+\frac{\rho^{2}}{n_{c}}\left(\frac{\partial v_{c}}{\partial \rho}\right)_{T, \mu_{c}=0}\right],
\end{aligned}
$$

where $N_{c}^{\mathrm{eq}}$ is the number of lattice sites such that the system remains at equilibrium and where the partial derivatives are evaluated around an equilibrium state point. Note that a Maxwell relation was used to reformulate the last pressure derivative. The virial contribution corresponds to a quenched system where particle rearrangements are not possible, so it is an upper bound to $B \equiv B_{\text {vir }}-B_{\text {corr }}$. The results for different state points are compared in Fig. 2 to the values obtained by direct numerical differentiation of the equilibrium free-energy curves. Remarkable agreement is obtained between the two approaches. Also, far from negligible, $B_{\text {corr }}$ results in a $B$ about $40 \%$ smaller than $B_{\text {vir }}$, as can also be gathered from Table I. The leading term to the correction, the change in $\mu_{c}$ with density, suggests that deletion of lattice sites weakens the system's response to compression. The
TABLE I. Bulk modulus decomposition and the sound mode elastic and coupling constants for three different multiplyoccupied crystal state points.

\begin{tabular}{ccccccccc}
\hline \hline$T$ & $\rho$ & $B$ & $B_{\text {vir }}$ & $B_{\text {corr }}$ & $c_{11}$ & $c_{12}$ & $c_{22}$ & $\omega$ \\
\hline 0.5 & 4.3 & 48.2 & 89.8 & 41.6 & 1.335 & 2.95 & 10.03 & 0.805 \\
0.8 & 6.2 & 100.5 & 177.1 & 76.6 & 1.346 & 3.05 & 15.35 & 0.670 \\
1.1 & 8.2 & 176.6 & 308 & 131.0 & 1.350 & 3.10 & 21.0 & 0.582 \\
\hline \hline
\end{tabular}

changes in lattice site occupancy permitted by particle overlap thus increase the crystal compressibility compared to simple affine transformations. Generally, this still translates into an increase of $B$ with density. Note also that the temperature dependence is rather weak. Varying $T$ from 0.5 to 1.1, the curves in Fig. 2 appear to collapse onto a master function, which suggests that entropic effects have little impact in this regime.

If sound waves have a period shorter than the time it takes for particles to redistribute between unit cells, then we can distinguish between two different sound modes in cluster solids. Density fluctuations stem either from changes to the unit cell volume $v_{c}$ at fixed cluster occupancy $n_{c}$ or from fluctuations in $n_{c}$ at fixed $v_{c}$, as schematized in Fig. 3. At constant $T$ and $V$, the free-energy density fluctuations $\Delta f \equiv \Delta F / V$ are then

$$
\Delta f=c_{11}\left(\rho_{c} \Delta n_{c}\right)^{2}+c_{22}\left(n_{c} \Delta \rho_{c}\right)^{2}+2 c_{12} \rho_{c} n_{c} \Delta n_{c} \Delta \rho_{c},
$$

where the elastic constants

$$
\begin{gathered}
c_{11}=\frac{1}{2 \rho_{c}}\left(\frac{\partial \mu}{\partial n_{c}}\right)_{\rho_{c}}, \quad c_{22}=\frac{1}{2 n_{c}^{2}}\left(\frac{\partial\left(\mu_{c}+\mu n_{c}\right)}{\partial \rho_{c}}\right)_{n_{c}}, \\
c_{12}=\frac{1}{2 \rho}\left(\frac{\partial\left(\mu \rho_{c}\right)}{\partial \rho_{c}}\right)_{n_{c}}
\end{gathered}
$$

can be obtained by numerically differentiating the simulation results. A change of variables to sound-mode space $x_{ \pm}=\left(\sqrt{c_{11}} \rho_{c} \Delta n_{c} \pm \sqrt{c_{22}} n_{c} \Delta \rho_{c}\right) / \sqrt{2}$, diagonalizes the expression

$$
\Delta f=(1+\omega) x_{+}^{2}+(1-\omega) x_{-}^{2}
$$

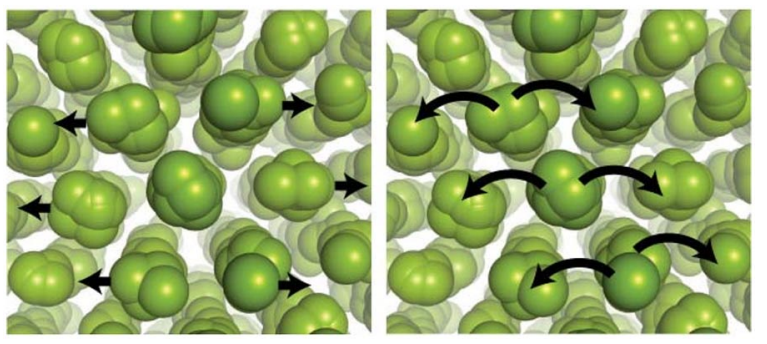

FIG. 3 (color online). Density fluctuations in multiple occupancy crystals either stem from (left) fluctuations in the unit cell volume or from (right) rearrangements in particle distributions between lattice sites. 
with coupling constant $\omega \equiv c_{12} / \sqrt{c_{11} c_{22}}$. The elastic coefficients and the coupling constant are presented for three different state points in Table I. Though these results are insufficient to paint the full physical picture, a couple of comments are in order. First, the $c_{11}$ term corresponds to the hopping of particles and is expected to be heavily damped, while the $c_{22}$ term is the equivalent of a longitudinal sound wave, which will propagate for long wavelengths. Second, for the temperature and density range under study, the first constant and the cross term increase only very little with $T$ and $\rho$, while the $c_{22}$ more than doubles. The increased density from one state point to the next is most certainly responsible for that, since higher temperatures would tend instead to facilitate lattice spacing fluctuations for a constant repulsive energy barrier. To the best of our knowledge, no theoretical predictions exist with which to further compare these results.

Starting from the formalism developed by Swope and Andersen [1], we have presented how simulations and experiments of multiple-occupancy crystals critically depend on the chemical potential associated with the insertion of a lattice site. Taking this into account within simulations allows for the precise determination of the equilibrium phase diagram of cluster crystals, which is much more subtle than for the traditional, singleoccupancy sort. Also, even though the chemical potential associated with lattice site insertion is strictly zero in equilibrium, its constrained derivatives are not. This has considerable impact on the calculation of the bulk modulus and the two sound modes' elastic constants, for example. Departing more drastically from thermodynamics, longlived nonequilibrium structures of cluster-crystal forming dendrimers might even be observable in rapidly quenched experimental systems, if the resulting ordered solids happen to end up in states with $\mu_{c} \neq 0$. These metastable crystals would then undergo phase transitions at different state points than those predicted by equilibrium thermodynamics. Finally, the generalization of the free-energy and elastic constant calculation methodology presented here has a broader applicability than for multiply-occupied crystals. It is the appropriate way to simulate systems with hyperlattices in general, such as in the quantum Hall effect as well as for certain liquid crystal phases, micellar crystals, or microphase separated colloids.

We would like to thank D. Gottwald, G. Kahl, C. N. Likos, M. Neumann, and B. Widom for helpful discussions. The work of the FOM Institute is part of the research program of FOM and is made possible by financial support from the Netherlands Organization for Scientific Research (NWO). Moreover, this work was carried out under the HPC-EUROPA Project (No. RII3-CT-2003-506079), with the support of the European Community-Research Infrastructure Action of the FP6. Computer time at the Dutch center for high-performance computing SARA is gratefully acknowledged. P.C. further acknowledges No. MIF1-CT-2006-040871 (EU) funding and B. M. M. acknowledges financial support by the Österreichische Forschungsfond (FWF) under Project No. P17823-N08 as well as travel funding by COST-P13 (MOLSIMU) and the Österreichische Forschungsgemeinschaft.

*frenkel@amolf.nl

[1] W. C. Swope and H. C. Andersen, Phys. Rev. A 46, 4539 (1992).

[2] P. A. Monson and D. A. Kofke, in Advances in Chemical Physics, edited by I. Prigogine and S. A. Rice, Advances in Chemical Physics Vol. 115 (John Wiley \& Sons, New York, 2000), pp. 113-179.

[3] S. Pronk and D. Frenkel, J. Phys. Chem. B 105, 6722 (2001).

[4] P. G. De Gennes and J. Prost, The Physics of Liquid Crystals, International Series of Monographs on Physics Vol. 83 (Clarendon Press, Oxford, 1993).

[5] M. M. Fogler, A. A. Koulakov, and B. I. Shklovskii, Phys. Rev. B 54, 1853 (1996).

[6] R. M. Lewis, Y. Chen, L. W. Engel, D. C. Tsui, P. D. Ye, L. N. Pfeiffer, and K. W. West, Phys. Rev. Lett. 93, 176808 (2004).

[7] C. N. Likos, A. Lang, M. Watzlawek, and H. Löwen, Phys. Rev. E 63, 031206 (2001).

[8] B. M. Mladek, D. Gottwald, G. Kahl, M. Neumann, and C. N. Likos, Phys. Rev. Lett. 96, 045701 (2006).

[9] C. N. Likos, B. M. Mladek, D. Gottwald, and G. Kahl, J. Chem. Phys. 126, 224502 (2007).

[10] H. Fragner, Phys. Rev. E 75, 061402 (2007).

[11] H. Fragner, Comput. Phys. Commun. 176, 327 (2007).

[12] B. M. Mladek, D. Gottwald, G. Kahl, M. Neumann, and C. N. Likos, J. Phys. Chem. B 111, 12799 (2007).

[13] B. M. Mladek, G. Kahl, and C. N. Likos, Phys. Rev. Lett. (to be published).

[14] D. Frenkel and B. Smit, Understanding Molecular Simulation, Computational Science Series Vol. 1 (Academic Press, San Diego, 2002).

[15] J.W. Gibbs, The Scientific Papers of J. Willard Gibbs, Thermodynamics Vol. 1 (Ox Bow Press, Woodbridge, 1993).

[16] A. J. Moreno and C. N. Likos, Phys. Rev. Lett. 99, 107801 (2007).

[17] A. Z. Panagiotopoulos, J. Chem. Phys. 112, 7132 (2000).

[18] D. Frenkel and A. J.C. Ladd, J. Chem. Phys. 81, 3188 (1984).

[19] B. M. Mladek, P. Charbonneau, and D. Frenkel (unpublished).

[20] B. Widom, J. Chem. Phys. 39, 2808 (1963).

[21] A.Z. Panagiotopoulos, Mol. Simul. 9, 1 (1992).

[22] J. Rowlinson, Liquids and Liquid Mixtures, Modern Aspects Series of Chemistry (Butterworths Scientific Publications, London, 1959).

[23] P. S. Y. Cheung, Mol. Phys. 33, 519 (1977). 